\title{
Analysis on a Preparation and Optical performance of Porous Silicon - Based Sensing Materials
}

\author{
Ran Zhang, Tiecheng Wang, Yonglei Zhao \\ TangShan Polytechnic College, Tangshan, Hebei, 063200
}

Keywords: Porous Silicon; Preparation; Optical performance; Sensing

\begin{abstract}
In the paper, the surface uniform porous silicon thin film material was prepared by using the pulse electrochemical etching method under orthogonal parameters. Analysis of Surface Morphology of Porous Silicon under Atomic Force Microscopy, the optimum preparation conditions were obtained with good optical properties. The optical reflectance spectra of porous silicon films with different porosities were obtained by using the designed porous silicon thin film reflection spectroscopy system, and the refractive index and absorption system spectra were obtained by MATLAB software simulation, which provides the basis for the development and application of porous silicon-based sensing devices.
\end{abstract}

\section{Introduction}

Porous silicon is a new optoelectronic material, because of its optoelectronic devices and in the field of sensing has a huge potential application value and much attention. However, the research of porous silicon-based sensing devices is in the exploratory stage, and the sensing performance needs to be further improved. In order to produce practical porous silicon-based sensing devices, it is necessary to understand the optical sensing properties of porous silicon-based thin films. At present, the research and practice of the formation mechanism, luminescence mechanism, optical properties and sensing properties of the porous silicon-based thin film materials have been progressively and gradually become unified. The refractive index of porous silicon-based thin film materials is an important parameter to design novel optical gas sensor. The surface morphology of porous silicon is also a key factor to determine its industrial application value. Therefore, this paper focuses on the analysis of porous silicon the experimental preparation of the sensory materials and the optical properties under different conditions.

\section{Preparation of Porous Silicon Based Thin Film Materials}

In this paper, the preparation of porous silicon material was carried out by electrochemical corrosion method in comparison with various preparation methods and analysis of the advantages and disadvantages.

Preparation of Porous Silicon-Based Thin Film Material Experimental Device Design. In this paper, the use of LabVIEW software to generate pulse information, the software to control the pulse signal duty cycle, corrosion time, current density and other corrosion parameters, so the experimental repeatability is guaranteed. Figure 1 is a schematic representation of a porous silicon preparation system.

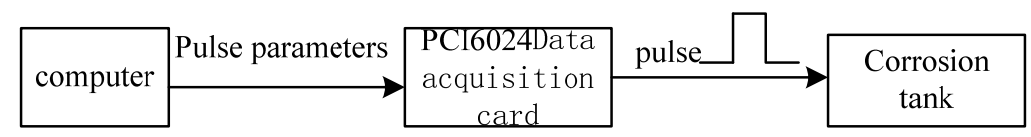

Fig.1 Schematic diagram of a porous silicon preparation system

(1) Computer. It is by the programming language LabVIEW, generate control procedures to generate a certain duty cycle and pulse cycle pulse signal, through the data acquisition channel to the PCI6024 data acquisition card. Experimental Labview generated pulse signal interface shown in Figure 2. 


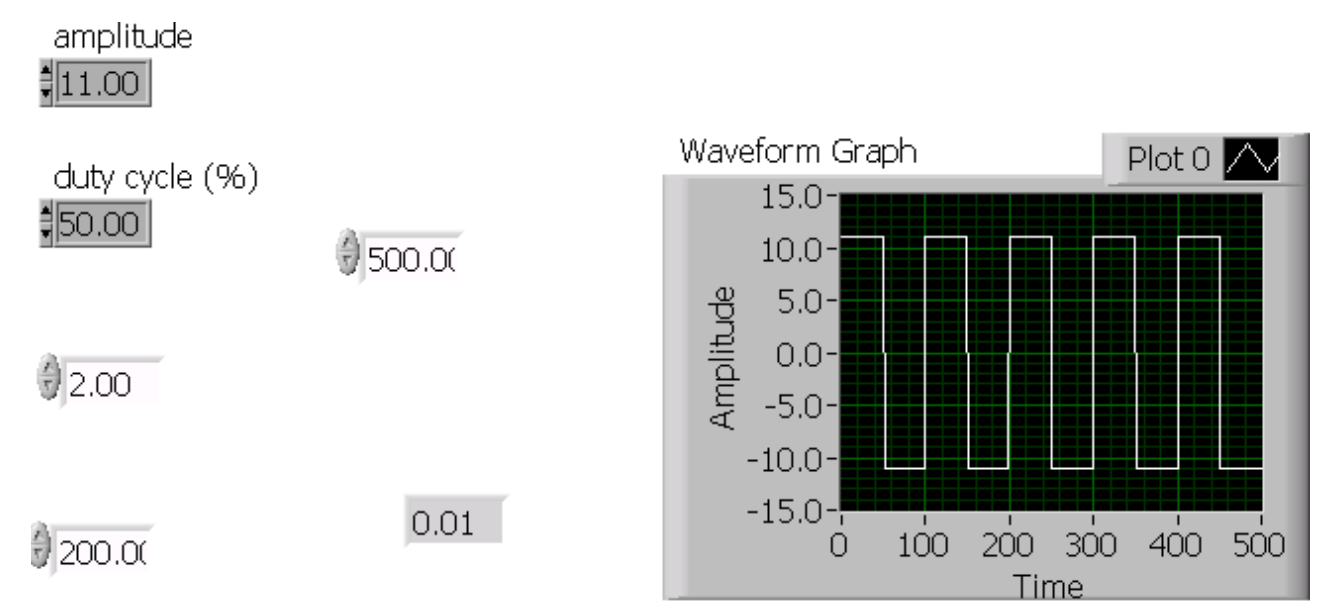

Fig.2 Experimental Labview generated pulse signal interface

(2) Data acquisition card. It acts as a corrosive slot and the computer's communication interface, the LabVIEW generated pulse parameters, resulting in pulse voltage applied to the corrosion tank.

(3) Corrosion tank. The corrosion tank used in the experiment is to improve the traditional single-cell structure, easy to operate. Electrolytic cell for the cylinder of polytetrafluoroethylene, will be dug two semi-cylindrical, the middle does not get through, to bridge the two pools. A pool packed with saturated $\mathrm{KCl}$ solution (analytical grade), another pool equipped with $\mathrm{HF}$ and ethanol mixed solution. And the other end of the two electrodes are respectively connected with the analog input and analog output ports of the PCI-6024 data acquisition card, and the other ends of the two electrodes are respectively connected with the analog input and analog output ports of the PCI-6024 data acquisition card. Phase up since the double-cell structure eliminates the interface potential, this device contributes to the preparation of porous silicon with uniform surface properties and excellent properties.

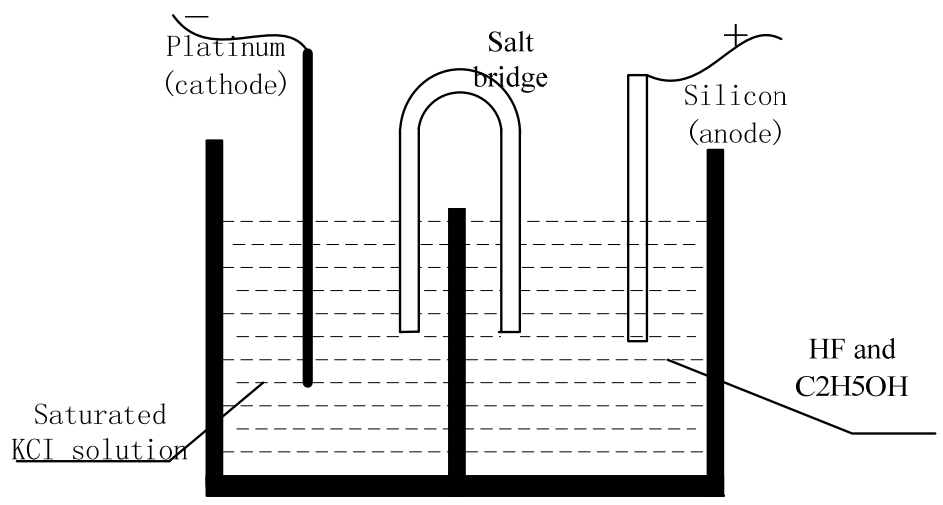

Fig. 3 Illustration

Preparation of Porous Silicon-Based Thin Film Material Experimental Parameters Set. Experiments using Labview software to generate pulse series, pulse width and pulse period (pulse width and idle time sum) is called the duty cycle. The effective electrochemical etch time is the same when preparing porous silicon samples with different cycles and duty cycles. The optical properties of porous silicon depend on the ratio of the etching solution, the current density, the pulsed electrochemical cycle and the duty cycle, and the etching time. After repeated experiments, the volume ratio of HF and ethanol in the etching solution was 1: 1, the anodic oxidation time was $15 \mathrm{~min}$, and the change of the pulse period, the duty cycle and the current density were calculated by the pulsed electrochemical corrosion method. The experimental parameters are shown in Table 1, and the corresponding orthogonal tables are shown in Table 2. 
Table 1 Preparation of porous silicon parameter setting table

\begin{tabular}{l|l|l|l}
\hline Level & Current density / $\mathrm{mA} \mathrm{cm}^{-2}$ & Duty cycle & Pulse cycle / ms \\
\hline 1 & 12 & $1 / 10$ & 10 \\
\hline 2 & 20 & $5 / 10$ & 50 \\
\hline 3 & 30 & $7 / 10$ & 1 \\
\hline
\end{tabular}

Table 2 Preparation of Porous Silicon Parameter Setting Orthographic Table

\begin{tabular}{l|l|l|l}
\hline Sample number & Current density $/ \mathrm{mA} \mathrm{cm}^{-2}$ & Duty cycle & Pulse cycle $/ \mathrm{ms}$ \\
\hline 1 & 12 & $1 / 10$ & 1 \\
\hline 2 & 12 & $5 / 10$ & 10 \\
\hline 3 & 12 & $7 / 10$ & 50 \\
\hline 4 & 20 & $1 / 10$ & 10 \\
\hline 5 & 20 & $5 / 10$ & 50 \\
\hline 6 & 20 & $7 / 10$ & 1 \\
\hline 7 & 30 & $1 / 10$ & 50 \\
\hline 8 & 30 & $5 / 10$ & 1 \\
\hline 9 & 30 & $7 / 10$ & 10 \\
\hline
\end{tabular}

Preparation of Porous Silicon-Based Thin Film Material Preparation Process. The experimental silicon wafer is a p-type single crystal with a resistivity of 0.03 to $0.05 \Omega \cdot \mathrm{cm}$ and a $<100>$ crystal orientation, and a thickness of $3 \mathrm{~mm}$. Before the experiment, the room temperature control in advance at $20{ }^{\circ} \mathrm{C}$, and keep the room clean, the humidity as far as possible to maintain a constant, the experimental use of the container with deionized water clean and dry preservation. The monocrystalline silicon is then pretreated.

(1) Test dicing monolithic silicon wafer was tested for $2 \mathrm{~cm} \mathrm{x} 2 \mathrm{~cm}$.

(2) Cleaning, aluminizing, vacuum quenching and sealing wax first, according to the deionized water - ethanol (analytical grade) - acetone (analytical grade) - ethanol - deionized water in the order of each step $4 \sim 5$ min ultrasonic cleaning, Drying. The main purpose of cleaning is to remove the grease in the wafer manufacturing process; and then steaming a layer of aluminum on the non-polished surface electrode, this step in the DMX-220 vacuum coating machine to complete; and then in a vacuum state of the Al layer was rapidly annealed at $420^{\circ} \mathrm{C}$ for $20 \mathrm{~min}$ to obtain good ohmic contact. Quenching is done in a two-room ultra-high vacuum annealing furnace. After quenching is completed, the entire non-polished and polished surfaces are not corroded, and the exposed electrodes are sealed with wax, keeping the porous area constant at $1 \mathrm{~cm} 2$.

(3) Drying and drying preservation Wrap the cleaned wafers and place them in a dry, clean surface dish for use in the test. After the preparation work is completed, the preparation is carried out according to the system shown in Fig. The prepared porous silicon sample is rapidly immersed in the $\mathrm{KOH}$ solution at a concentration of 3\% after being taken out from the etching tank and the prepared porous silicon film is peeled off from the silicon substrate. The porous silicon film was then rinsed with deionized water and dried with a stream of nitrogen. Finally, the surface morphology of porous silicon was analyzed, and the optical reflectance spectra of porous silicon-based thin films were measured on the experimental platform. The optical constants and optical properties were also analyzed. 


\section{Optical Properties of Porous Silicon-Based Thin Film Measurement System}

The reflection spectrum measurement system of the porous silicon-based thin film material is composed of a light source, a fiber bundle, an isolator, a reflective fiber optic probe, a base, a coupling lens, a small spectrometer and a personal computer. The working principle and the selected model of each component are explained in turn.

(1) Broadband light source using Japan's Ando's AQ4305 white light source, the output wavelength of $400 \mathrm{~nm}-1800 \mathrm{~nm}$; $-40 \mathrm{dBm}$ (bandwidth $10 \mathrm{~nm}$ ); free space output; stability $0.05 \mathrm{~dB}$; provide broadband light source.

(2) Spectrometer Japan Ando company's spectrum analyzer, model AQ-6317B, wavelength resolution of $0.2 \mathrm{~nm}$, wavelength accuracy of $0.02 \mathrm{~nm}$, dynamic range of $60 \mathrm{~dB}$.

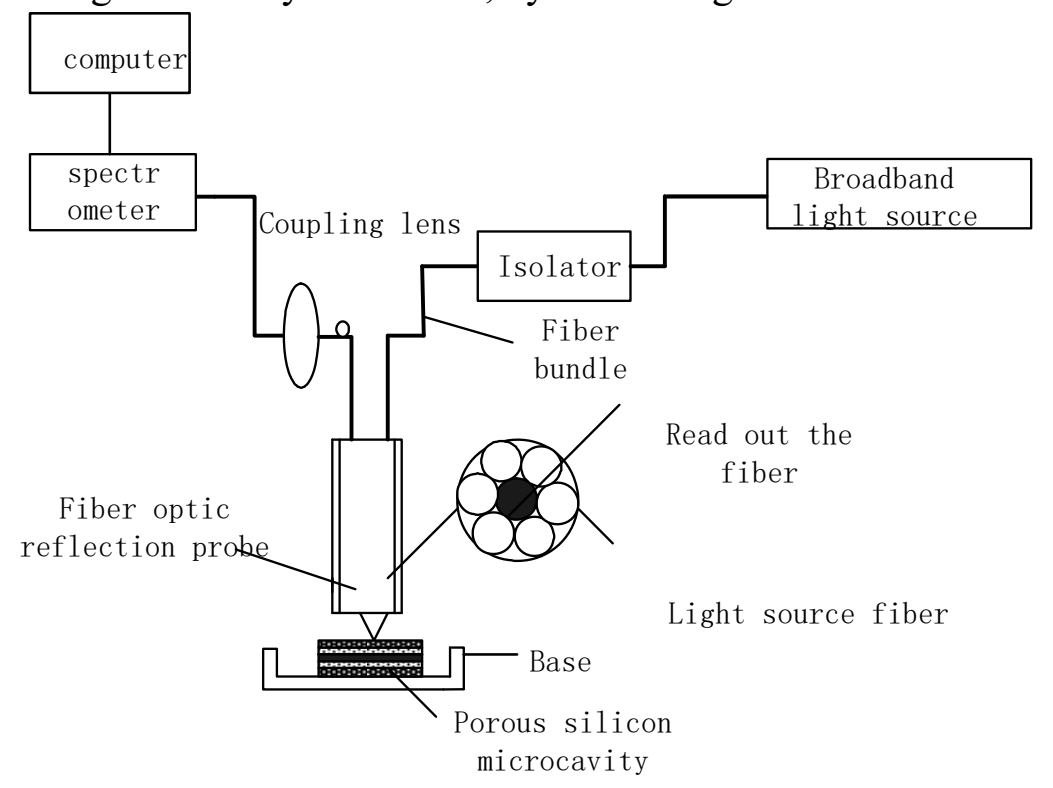

Figure 4 Schematic diagram of the reflectance measurement system for porous silicon thin film materials

(3) Reflective fiber optic probe Reflective optical fiber sensor through the object from the surface of the fiber and optical fiber to accept the changes in the optical signal to detect the information on the reflective surface parameters. Because the reflective fiber probe in this experimental system is not used for sensing measurement, but for the light of the receiving and output, compare the advantages and disadvantages of a variety of arrangement, coaxial output of the strongest signal intensity, and loss Minimum, so choose coaxial reflective fiber optic probe.

(4) Fiber optic beam selection of optical fiber for the measurement, the technical indicators are as follows: Park / square -0.2; Park / line -0.2; Park / Park -0.2; special specifications can be customized; optical fiber transmittance $\geq 60 \%$; Spectral range: $0.19-2.00 \mathrm{~nm}$; mainly used for spectral analysis, lighting, sensing, fluorescence detection and other fields.

\section{Analysis of Surface Morphology and Optical Properties of Porous Silicon Substrate Films}

Analysis of Surface Morphology of Porous Silicon Based Thin Films. The surface morphology of the porous silicon thin film prepared by orthogonal force microscopy (AFM) was fabricated by SOLVER atomic force microscope. The model was P47 with a resolution of about $2 \mathrm{~nm}$ and a curvature of the probe tip the radius is about $10 \mathrm{~nm}$.

The prepared porous silicon film was peeled off from the silicon substrate at a concentration of $3 \% \mathrm{KOH}$ solution. The prepared porous silicon samples will exhibit different surface luster, such as yellowish, brown and orange, depending on the degree of corrosion. The surface morphology of porous silicon was observed by AFM at room temperature. 5 (a) to (h) show the AFM surface 
topography of porous silicon samples prepared under various electrochemical corrosion parameters. The porosities of the eight samples were $11.2 \%, 51.3 \%, 39.0 \%, 67.2 \%, 69.9 \%, 78.4 \%, 58.0 \%$ and $56.7 \%$ respectively by weighing method.

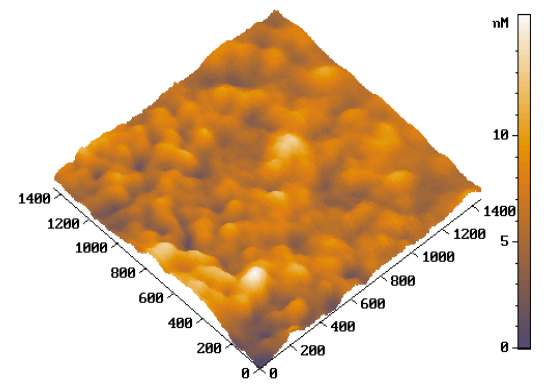

(a) Sample 1

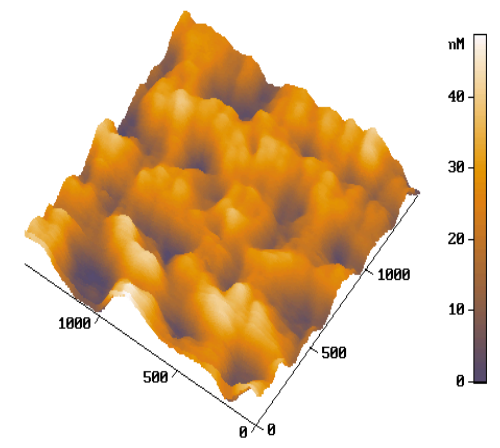

(c) Sample 7

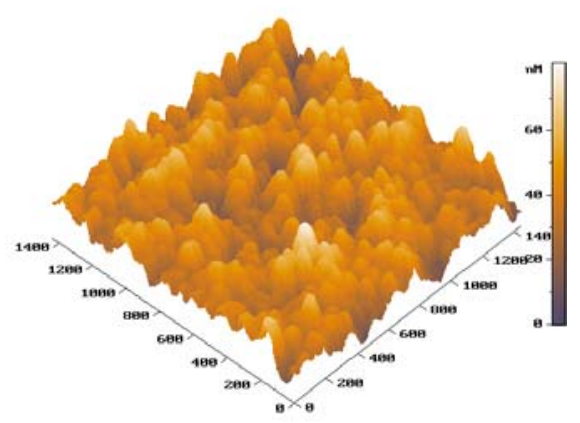

(e) Sample 5

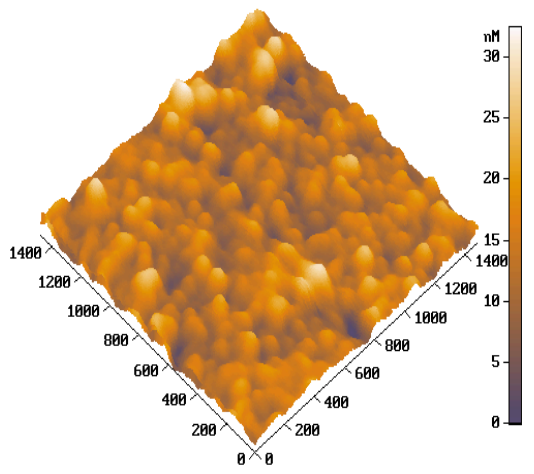

(b) Sample 4

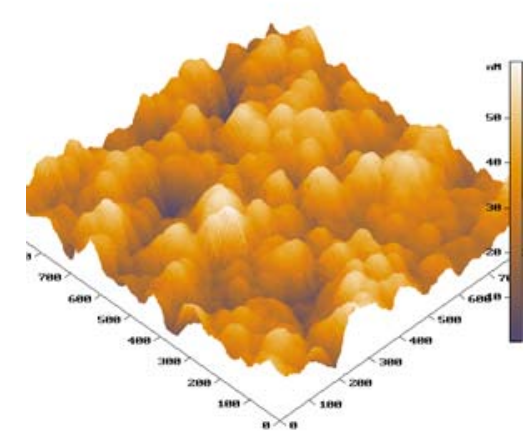

(d) Sample 8

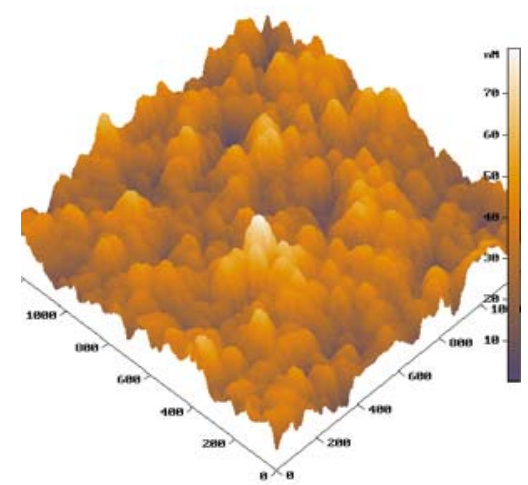

(f) Sample 2 


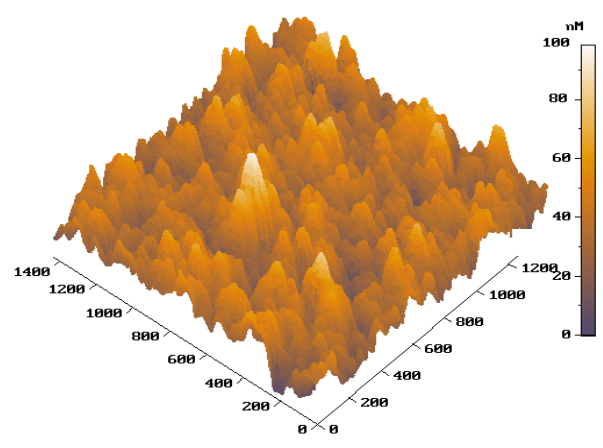

(g) Sample $y$

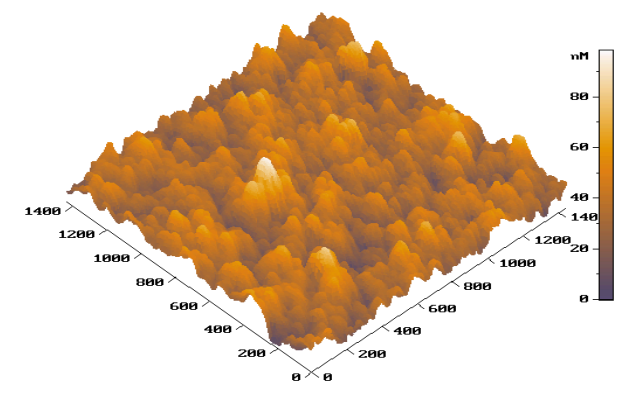

(h) Sample 6

Figure 5 AFM surface morphology of porous silicon prepared in orthogonal experiment (unit: $\mathrm{nm})$

The porosities of the eight samples were $11.2 \%, 51.3 \%, 39.0 \%, 67.2 \%, 69.9 \%, 78.4 \%, 58.0 \%$ and $56.7 \%$ respectively.

Figure $5(\mathrm{a}) \sim(\mathrm{c})$ corresponds to the duty cycle of $1 / 10$, the effective corrosion time of $15 \mathrm{~min}$, current density and pulse cycle vary. Compared with other topography, the holes in (a) to (c) are shallow, and the roots of the holes are densely connected together. In particular, (a) the monocrystalline silicon surface is hardly corroded, and only the edges are sparsely distributed in several pores with very uneven pores. Figure (c) corresponds to the AFM map, although relatively independent, but the aperture close to $200 \mathrm{~nm}$, does not meet the conditions of quantum confinement luminescence, which can be inferred luminescence intensity will be weak. It can be seen: the greater the current density, the greater the pulse period, the greater the aperture. In the graphs (d) to (f), the distribution of the pores is uniform, the characteristics of the roots are "isolated" and "the protrusions" are remarkable, the surface roughness is high, and the average depth of the pores reaches $70 \mathrm{~nm}$, the pore size is about $20 \sim 50 \mathrm{~nm}$ Between the better performance. Figure $(\mathrm{g}) \sim(\mathrm{h})$ corresponding to the duty cycle are $7 / 10$. It can be seen that the average size of the hole is the deepest, but the surface of the porous silicon thin film is clearly localized, the surface is not smooth and smooth. From the results analysis, it can be seen that when the duty ratio is $5 / 10$, the prepared porous silicon specimen surface is more uniform, the optical performance is more stable and the quality is the best.

It was also found that the diameter of the silicon column increased with the increase of the anodic oxidation time, and the thickness of the film increased, but the anodic oxidation time had no effect on the porosity of the porous silicon. The current density, the increase in the diameter of the silicon column increases the HF acidity, the porosity decreases and the silicon column size increases.

Optical Properties of Porous Silicon-Based Thin Films. The reflection spectrum of the prepared porous silicon sample was measured by using the reflection device shown in Fig. 4, and the experimental results were analyzed. Figure 7 shows the reflectance spectra of porous silicon samples with $67.2 \%, 56.7 \%, 78.4 \%$ and $69.9 \%$ porosity, respectively, in the visible wavelength range. 


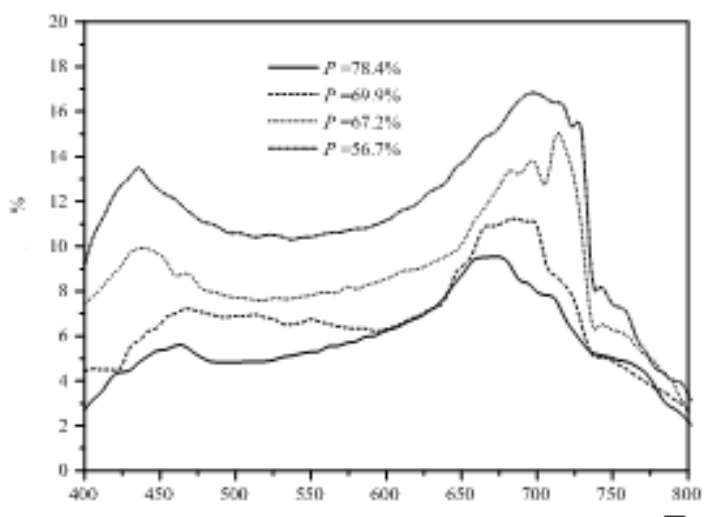

Figure 6 Normalized reflection spectrum of porous silicon

In Fig. 6, it can be seen that the increase of the porosity leads to the decrease of the reflectivity. The reflection spectrum of the porous silicon is irregular and the reflection curve has no uniform function expression. However, the reflection spectrum of the porous silicon sample with different porosity is the same as the incident wavelength The reflectivity is very low, less than $20 \%$; the reflection spectrum is between $432.9 \sim 467.8 \mathrm{~nm}$ (lower reflection peak) and $675 \sim 705$ (the lower reflection peak), and the reflection rate is very low, $\mathrm{Nm}$ (higher reflection peak), and the lower the porosity, the more obvious the peak phenomenon is due to the porous structure of porous silicon; with the increase of porosity, the lower The reflection peak exhibits a blue shift trend, while the higher reflection peak moves in the long wave direction, and the distance (bandwidth) between the two peaks increases. The reflection spectrum of monocrystalline silicon exhibits multiple reflection peaks, and as a semiconductor material, the reflectivity is high, reaching 75\%. At $615 \mathrm{~nm}$, there is a narrow peak with a half-peak width and more porous silicon. It can be seen from Figure 7 that the reflectivity of porous silicon is much smaller than that of monocrystalline silicon and retains the properties of a part of monocrystalline silicon. The local fluctuation of porous silicon in the visible region is larger than that of single crystal silicon.

According to the measurement curve of the reflection spectrum of porous silicon, the corresponding Matlab program is written to obtain the relationship between the refractive index and the absorption coefficient of porous silicon with the wavelength and porosity of the incident light. The refractive index spectrum of the porous silicon thin film and the absorption coefficient of the porous silicon thin film are shown in Fig. 7 and Fig. 8.

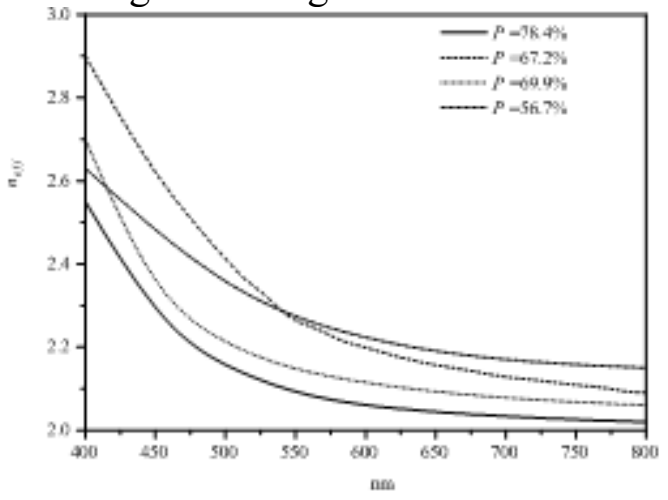

Figure 7 Refractive index spectra of porous silicon films 


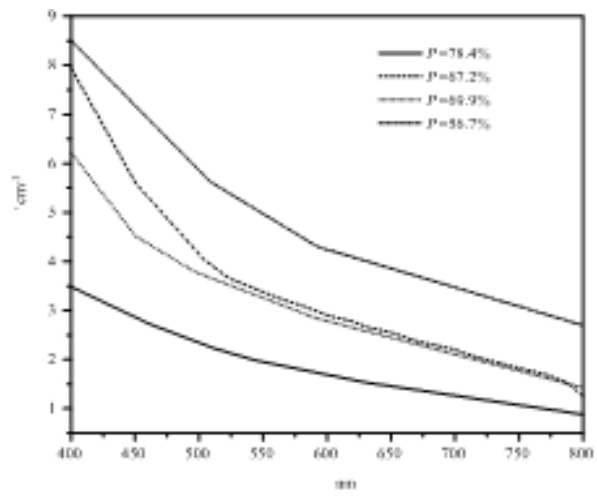

Figure 8 Absorption coefficient of porous silicon film

In theory, the effective refractive index of porous silicon is between 1 and 3.4, and the refractive index of the porous silicon samples with different porosities in the visible range is between 2.0 and 2.9. In the refractive index spectrum, the size of the refractive index of the porous silicon in the initial wavelength is uncertain, but in the visible edge region, the porous silicon sample with large porosity has a small effective refractive index.

The absorption coefficient of porous silicon decreases with the increase of the incident wavelength, and the larger the porosity is, the smaller the absorption coefficient is, and the larger the porosity is, the smoother the curve is. The change in the absorption coefficient of the porous silicon is proportional to the extinction coefficient, but the value of the absorption coefficient is much smaller. The absorption coefficient of the visible light band is between $1 \times 10-3 \sim 9 \times 10-3$ $\mathrm{cm}-1$.

\section{Conclusion}

A porous silicon film was prepared by pulsed electrochemical etching. The results show that the porous silicon surface is "undulating" on the surface of the porous silicon, the best preparation conditions (duty ratio 1: 1, current density of $12 \mathrm{~mA} \mathrm{~cm}-2$, pulse period of $10 \mathrm{~ms}$ ), Silicon column diameter between $20 \sim 40 \mathrm{~nm}$, the surface smoothness, good optical properties. The reflection spectrum of porous silicon thin film material is irregular and the reflectivity is very low. There are two reflection peaks in the visible region, and the peak value is different, and the reflection peak is between 675 and $705 \mathrm{~nm}$. With the increase of porosity, the lower reflection peak showed a blue shift trend, while the higher reflection peak shifted to the long wave direction, and the distance (bandwidth) between the two peaks increased. The refractive index, extinction coefficient, the coefficients and the dielectric function spectrum decrease with the increase of porosity and incident wavelength. The absorption coefficient of porous silicon in the visible light band is very small, which is based on porous silicon-based thin film materials, the development and application of new gas sensors laid the foundation.

\section{Acknowledgements}

Fund Project: Hebei Province higher school scientific research project "Research and design of a new type of optical gas sensor" (Z2015203)

\section{References}

[1] Zhang Hua, Li Jiao, from the days of Min. Research and Application of Porous Silicon[J]. Journal of Shandong University of Technology. 2015, 29 (3): 20-24.

[2] Mo Ruihai, Liu Hongli, Zhang Xiong. Study on Preparation Conditions of Porous Silicon for Photonic Crystals[J]. Semiconductor Optoelectronics, 2013, 34 (5): 811-814

[3] Tian Jincheng, Yang Zhiwei. Electroluminescence Study of Porous Silicon[J]. Journal of Qingdao University, 2015, 28 (2): 22-24. 
[4] Chen Ying, Han Yangyang, Cao Huiying, Zhu Qiguang, Bi Weihong. Refractive index sensing properties of mirror symmetric porous silicon photonic crystals[J]. Chinese laser, 2016, 43 (4): $120-126$. 